\title{
The perimeter of a flattened ellipse can be estimated accurately even from Maclaurin's series
}

\author{
VITO LAMPRET \\ University of Ljubljana, 386 Slovenia \\ vito. lampret@guest.arnes.si
}

\begin{abstract}
For the perimeter $P(a, b)$ of an ellipse with the semi-axes $a \geq b \geq 0$ a sequence $Q_{n}(a, b)$ is constructed such that the relative error of the approximation $P(a, b) \approx Q_{n}(a, b)$ satisfies the following inequalities

$$
\begin{aligned}
0 \leq-\frac{P(a, b)-Q_{n}(a, b)}{P(a, b)} & \leq \frac{\left(1-q^{2}\right)^{n+1}}{(2 n+1)^{2}} \\
& \leq \frac{1}{(2 n+1)^{2}} e^{-q^{2}(n+1)},
\end{aligned}
$$
\end{abstract}

true for $n \in \mathbb{N}$ and $q=\frac{b}{a} \in[0,1]$.

\section{RESUMEN}

Para el perímetro $P(a, b)$ de una elipse con semiejes $a \geq b \geq 0$, se construye una sucesión $Q_{n}(a, b)$ tal que el error relativo de la aproximación $P(a, b) \approx Q_{n}(a, b)$ satisface las siguientes desigualdades

$$
\begin{aligned}
0 \leq-\frac{P(a, b)-Q_{n}(a, b)}{P(a, b)} & \leq \frac{\left(1-q^{2}\right)^{n+1}}{(2 n+1)^{2}} \\
& \leq \frac{1}{(2 n+1)^{2}} e^{-q^{2}(n+1)},
\end{aligned}
$$

válidas para $n \in \mathbb{N}$ y $q=\frac{b}{a} \in[0,1]$.

Keywords and Phrases: approximation, elementary, ellipse, estimate, Maclaurin series, mathematical validity, perimeter, simple.

2010 AMS Mathematics Subject Classification: 40A25, 65B10. 


\section{Introduction}

Injective parametric equations of the border of an ellipse having semi-axes of lengths $a$ and $b \leq a$ are given as $x=x(t)=a \cos (t), y=y(t)=b \sin (t)$, where $t \in[0,2 \pi)$. Its perimeter $P(a, b)$ is determined as

$$
\begin{aligned}
P(a, b) & =\int_{0}^{2 \pi} \sqrt{\dot{x}^{2}(t)+\dot{y}^{2}(t)} \mathrm{d} t=4 \int_{0}^{\frac{\pi}{2}} \sqrt{a^{2} \sin ^{2}(t)+b^{2} \cos ^{2}(t)} \mathrm{d} t \\
& =4 a \int_{0}^{\frac{\pi}{2}} \sqrt{1-\epsilon^{2} \cos ^{2}(t)} \mathrm{d} t \overbrace{t=\pi / 2-\tau}^{=} 4 a \int_{\frac{\pi}{2}}^{0} \sqrt{1-\epsilon^{2} \sin ^{2}(\tau)}(-\mathrm{d} \tau) .
\end{aligned}
$$

Thus, the perimeter $P(a, b)$ of an ellipse having semi-axes of lengths $a$ and $b \leq a$, is given as

$$
P(a, b)=4 a E(\epsilon)
$$

where

$$
E(\epsilon):=\int_{0}^{\frac{\pi}{2}} \sqrt{1-\epsilon^{2} \sin ^{2}(\tau)} \mathrm{d} \tau
$$

is complete elliptic integral of the second kind and

$$
\epsilon:=\sqrt{1-\left(\frac{b}{a}\right)^{2}}=\sqrt{\frac{a^{2}-b^{2}}{a^{2}}} \in[0,1),
$$

is the eccentricity of an ellipse.

For $b \approx 0$ it is intuitively evident that $P(a, b)>2 \times 2 a=4 a$. Moreover, since the functions $\epsilon \mapsto 1-\epsilon^{2} \sin ^{2}(\tau)$ are decreasing on the interval $[0,1]$ for any $\tau \in[0, \pi / 2]$, the function $E(\epsilon)$ is decreasing too. Therefore, we have

$$
1=\int_{0}^{\frac{\pi}{2}} \cos (\tau) \mathrm{d} \tau=E(1) \leq E(\epsilon) \leq E(0)=\frac{\pi}{2}
$$

for $0 \leq \epsilon \leq 1$. Consequently, due to (1.1),

$$
\inf _{0<b \leq a} P(a, b)=4 a<P(a, b) \leq P(a, a)=2 a \pi .
$$

The first exact formula for an ellipse perimeter was presented 277 years ago by Collin Maclaurin [24], given as the sum of infinite series:

$$
\begin{aligned}
P(a, b) & =2 \pi a \sum_{k=0}^{\infty}\left(\begin{array}{c}
\frac{1}{2} \\
k
\end{array}\right)^{2}(1-2 k) \epsilon^{2 k} \\
& =2 \pi a \sum_{k=0}^{\infty}\left(\frac{(2 k) !}{\left(2^{k} k !\right)^{2}}\right)^{2} \frac{\left(-\epsilon^{2 k}\right)}{2 k-1} \\
& =2 \pi a\left\{1-\sum_{k=0}^{\infty}\left[\frac{1}{4^{k}}\left(\begin{array}{c}
2 k \\
k
\end{array}\right)\right]^{2} \frac{\epsilon^{2 k}}{2 k-1}\right\},
\end{aligned}
$$

valid for $0 \leq \epsilon \leq 1$, where $\epsilon=\left(1-b^{2} / a^{2}\right)^{1 / 2}$, called the eccentricity ${ }^{1}$ of an ellipse. This series

\footnotetext{
${ }^{1}$ We have $\epsilon=\sqrt{1-q^{2}}$, where $q:=b / a$ is called the aspect ratio of an ellipse.
} 
originates from the integral (1.2). Later, Ivory [13] discovered a faster converging series for the integral (1.2), which was later significantly improved by Gauss and Kummer. Additionally, Gauss developed very efficient, swiftly convergent method of arithmetic-geometric means for computation of the integral (1.2), see [1]. Subsequently, a lot of approximations of the ellipse perimeter have been found. For example, among them is very popular Ramanujan's "extraordinarily unusual and exotic" approximation [2]. Motivated by the Barnard-Pearce-Schovanec approximations [3] and Villarino's contribution on the accuracy of a Ramanujan's approximation [29] and his paper [28], we shall derive elementarily ${ }^{2}$ an asymptotic estimate of the ellipse perimeter, based on the oldest Maclaurin series expansion. The result obtained surpasses most of the previous approximations.

\section{Background}

\subsection{The binomial approximation}

Using Taylor's formula (see for example [15, p. 111] with $x_{0}=0, h=x$ and $p=n$ ),

$$
f(x)=f(0)+\sum_{i=1}^{n} \frac{f^{(i)}(0)}{i !} x^{i}+\frac{x^{n+1}}{n !} \int_{0}^{1}(1-t)^{n} f^{(n+1)}(t x) \mathrm{d} t
$$

(true for $a, b \in \mathbb{R}, a<b, n \in \mathbb{N}, x \in[a, b]$ and $\left.f \in C^{n+1}[a, b]\right)$ for the function $f(x) \equiv(1+x)^{\frac{1}{2}}$, we obtain $^{3}$

$$
\begin{aligned}
(1+x)^{\frac{1}{2}}=1 & +\sum_{i=1}^{n}\left(\begin{array}{c}
\frac{1}{2} \\
i
\end{array}\right) x^{i} \\
& +x^{n+1} \int_{0}^{1}(1-t)^{n}\left(\begin{array}{c}
\frac{1}{2} \\
n+1
\end{array}\right)(n+1)(1+t x)^{\frac{1}{2}-n-1} \mathrm{~d} t,
\end{aligned}
$$

valid for $x \in(-1,1]$ and $n \in \mathbb{N}$.

Introducing $w_{i}$, called the $i$-th Wallis ratio, for $^{4} i \geq 0$,

$$
w_{i}:=\prod_{j=1}^{i} \frac{2 j-1}{2 j}=\frac{(2 i) !}{4^{i}(i !)^{2}}=\frac{1}{4^{i}}\left(\begin{array}{c}
2 i \\
i
\end{array}\right)
$$

we obtain

$$
\begin{aligned}
& \left(\begin{array}{c}
\frac{1}{2} \\
i
\end{array}\right)=\frac{\prod_{j=0}^{i-1}\left(\frac{1}{2}-j\right)}{i !}=(-1)^{i-1} \frac{1}{2^{i}} \cdot \frac{\prod_{j=1}^{i-1}(2 j-1)}{\prod_{j=1}^{i} j} \\
& =(-1)^{i-1} \frac{1}{2 i-1} \prod_{j=1}^{i} \frac{2 j-1}{2 j}=\underline{\underline{(-1)^{i-1} \frac{w_{i}}{2 i-1}}} .
\end{aligned}
$$

\footnotetext{
${ }^{2}$ not using complex analysis and absolute and uniform convergence of a series, as was used, for example, in [18] ${ }^{3}$ considering the identity $f^{(i)}(x) \equiv\left(\begin{array}{c}\frac{1}{2} \\ i\end{array}\right)(i !)(1+x)^{\frac{1}{2}-i}$

${ }^{4} \prod_{j=m}^{n} x_{j}:=1$, for $m>n$; consequently $w_{0}=1$
} 
Thus, thanks to (2.1), replacing $x$ by $-x$, we get

$$
(1-x)^{\frac{1}{2}}=1-\sum_{i=1}^{n} \frac{w_{i}}{2 i-1} x^{i}+r_{n}(x)
$$

with the remainder

$$
r_{n}(x)=-x^{n+1} \frac{w_{n+1}}{2 n+1}(n+1) \int_{0}^{1}\left(\frac{1-t}{1-t x}\right)^{n} \frac{\mathrm{d} t}{(1-t x)^{\frac{1}{2}}},
$$

estimated, for $x \in(0,1)$, as

$$
\begin{aligned}
0<-r_{n}(x) & =\frac{x^{n+1}}{(1-x)^{\frac{1}{2}}} \cdot \frac{w_{n+1}}{2 n+1}(n+1) \int_{0}^{1}\left(\frac{1-t}{1-t x}\right)^{n} \mathrm{~d} t \\
& <\frac{w_{n+1}}{(1-x)^{\frac{3}{2}}(2 n+1)} x^{n+1} .
\end{aligned}
$$

Indeed, using the substitution $\tau=\frac{1-t}{1-t x}$, i.e. $t=\frac{1-\tau}{1-\tau x}$ we have (considering $x \in(0,1)$ )

$$
\begin{aligned}
\int_{0}^{1}\left(\frac{1-t}{1-t x}\right)^{n} \mathrm{~d} t & =\int_{1}^{0} \tau^{n}\left(-\frac{1-x}{(1-\tau x)^{2}}\right) \mathrm{d} \tau=\int_{0}^{1} \tau^{n} \cdot \frac{1-x}{(1-\tau x)^{2}} \mathrm{~d} \tau \\
& <\int_{0}^{1} \tau^{n} \cdot \frac{1-x}{(1-x)^{2}} \mathrm{~d} \tau=\frac{1}{(1-x)(n+1)}
\end{aligned}
$$

\section{$2.2 \quad$ Wallis ratios estimates}

The integrals

$$
I_{n}:=\int_{0}^{\frac{\pi}{2}} \sin ^{n}(x) \mathrm{d} x \quad(n \geq 0),
$$

satisfy the recurrence relation

$$
I_{n}=\frac{n-1}{n} I_{n-2}, \quad \text { for } n \geq 2,
$$

where, obviously, we have $I_{0}=\frac{\pi}{2}$ and $I_{1}=1$. Consequently, by induction we find

$$
I_{2 i}=\prod_{j=1}^{i} \frac{2 j-1}{2 j} \cdot \frac{\pi}{2}=w_{i} \cdot \frac{\pi}{2}
$$

and

$$
I_{2 i+1}=\prod_{j=1}^{i} \frac{2 j}{2 j+1}=\frac{1}{(2 i+1) w_{i}} .
$$

Obviously, we estimate

$$
0<\sin ^{2 i+2}(x)<\sin ^{2 i+1}(x)<\sin ^{2 i}(x)<1,
$$


for $x \in\left(0, \frac{\pi}{2}\right)$ and $i \in \mathbb{N}$. Integrating, we obtain

$$
0<I_{2 i+2}<I_{2 i+1}<I_{2 i}<1,
$$

for all $i \in \mathbb{N}$. Hence, thanks to (2.7)-(2.8), we get

$$
\frac{2 i+1}{2 i+2} w_{i} \cdot \frac{\pi}{2}=w_{i+1} \cdot \frac{\pi}{2}<\frac{1}{(2 i+1) w_{i}}<w_{i} \cdot \frac{\pi}{2} .
$$

Consequently,

$$
\frac{2}{\pi} \cdot \frac{1}{2 i+1}<w_{i}^{2}<\frac{2}{\pi} \cdot \frac{1}{2 i-1} \quad(i \in \mathbb{N}) .
$$

We remark that there exists a huge literature on useful, more accurate estimates for $w_{n}$, e.g. $[4,5,6,7,8,9,10,11,12,14,16,17,19,20,21,22,23,25,26,27,31]$. However, for our needs, there suffice rather rough estimates (2.9).

\subsection{Some logarithmic formula expansion}

For $p \geq 1$ and $-1<t<1$ we have

$$
\begin{aligned}
2 \sum_{j=0}^{p-1} t^{2 j} & =\sum_{k=0}^{2(p-1)}\left(t^{k}+(-t)^{k}\right)=\sum_{k=0}^{2(p-1)} t^{k}+\sum_{k=0}^{2(p-1)}(-t)^{k} \\
& =\frac{1-t^{2 p-1}}{1-t}+\frac{1-(-t)^{2 p-1}}{1+t} .
\end{aligned}
$$

Consequently, integrating from 0 to $x \in(-1,1)$, the first and the last part of these equalities, we obtain

$$
\begin{aligned}
2 \sum_{j=0}^{p-1} \frac{x^{2 j+1}}{2 j+1} & =\int_{0}^{x} \frac{1}{1-t} \mathrm{~d} t-\int_{0}^{x} \frac{t^{2 p-1}}{1-t} \mathrm{~d} t+\int_{0}^{x} \frac{1}{1+t} \mathrm{~d} t+\int_{0}^{x} \frac{t^{2 p-1}}{1+t} \mathrm{~d} t \\
& =-\ln (1-x)+\ln (1+x)-\underbrace{\int_{0}^{x}\left(\frac{1}{1-t}-\frac{1}{1+t}\right) t^{2 p-1} \mathrm{~d} t}_{=R_{p}^{*}(x)} .
\end{aligned}
$$

Thus,

$$
\ln \left(\frac{1+x}{1-x}\right)=2 \sum_{i=1}^{p} \frac{x^{2 i-1}}{2 i-1}+R_{p}^{*}(x),
$$

with the remainder $R_{p}^{*}(x)$,

$$
R_{p}^{*}(x):=\int_{0}^{x} \frac{2 t^{2 p}}{1-t^{2}} \mathrm{~d} t \geq \int_{0}^{x} 2 t^{2 p} \mathrm{~d} t . \quad(0<x<1),
$$

estimated as

$$
\frac{2 x^{2 p+1}}{2 p+1}<R_{p}^{*}(x)<\frac{2 x^{2 p+1}}{\left(1-x^{2}\right)(2 p+1)} \quad(p \in \mathbb{N}, 0<x<1)
$$


From (2.10)-(2.11) we end up with the expansion

$$
\ln \left(\frac{1+x}{1-x}\right)=2 \sum_{i=1}^{\infty} \frac{x^{2 i-1}}{2 i-1},
$$

true for $x \in(0,1)$ and, consequently, also for $x \in(-1,0]$.

\section{The Maclaurin series}

\subsection{Derivation}

Referring to (2.4)-(2.5) and (2.6)-(2.7), we have, for any $n \in \mathbb{N}$,

$$
\begin{aligned}
\int_{0}^{\frac{\pi}{2}} \sqrt{1-\epsilon^{2} \sin ^{2}(\tau)} \mathrm{d} \tau & =\frac{\pi}{2}-\sum_{i=1}^{n} \frac{w_{i} \epsilon^{2 i}}{2 i-1} \int_{0}^{\frac{\pi}{2}} \sin ^{2 i}(\tau) \mathrm{d} \tau+r_{n}^{*}(\epsilon) \\
& =\frac{\pi}{2}-\sum_{i=1}^{n} \frac{w_{i} \epsilon^{2 i}}{2 i-1}\left(w_{i} \frac{\pi}{2}\right)+r_{n}^{*}(\epsilon)
\end{aligned}
$$

Hence

$$
\int_{0}^{\frac{\pi}{2}} \sqrt{1-\epsilon^{2} \sin ^{2}(\tau)} d \tau=\frac{\pi}{2}\left(1-\sum_{i=1}^{n} \frac{w_{i}^{2}}{2 i-1} \epsilon^{2 i}\right)+r_{n}^{*}(\epsilon),
$$

where $w_{i}$ is the $i$-th Wallis' ratio and the error term $r_{n}^{*}(\epsilon):=\int_{0}^{\pi / 2} r_{n}\left(\epsilon^{2} \sin ^{2}(\tau)\right) \mathrm{d} \tau$ is estimated, due to (2.5) and considering (2.6)-(2.7), as

$$
\begin{aligned}
0 \leq-r_{n}^{*}(\epsilon) & \leq \frac{\epsilon^{2 n+2}}{1-\epsilon^{2}} \cdot \frac{w_{n+1}}{2 n+1} \int_{0}^{\frac{\pi}{2}} \sin ^{2 n+2}(\tau) \mathrm{d} \tau \\
& =\frac{\epsilon^{2 n+2} w_{n+1}}{\left(1-\epsilon^{2}\right)(2 n+1)} \cdot w_{n+1} \frac{\pi}{2} .
\end{aligned}
$$

Thus, according to (2.9),

$$
0 \leq-r_{n}(\epsilon) \leq \frac{\pi}{2} \cdot \frac{1}{1-\epsilon^{2}} \cdot \frac{w_{n+1}^{2}}{2 n+1} \epsilon^{2 n+2} \leq \frac{1}{1-\epsilon^{2}} \cdot \frac{\epsilon^{2 n+2}}{(2 n+1)^{2}} .
$$

This estimate is not usable for $\epsilon \approx 1$, i.e. for $b \approx 0$ (for a very flattened ellipse).

As $w_{n}^{2} \leq 1$, we have $\lim _{n \rightarrow \infty} r_{n}(\epsilon)=0$ for any $\epsilon<1$, which is always true for ordinary ellipse, due to the equivalence $\epsilon=1 \stackrel{n \rightarrow \infty}{\Leftrightarrow} b=0$. Hence, there holds the so-called Maclaurin series expansion ${ }^{5}$

$$
\int_{0}^{\frac{\pi}{2}} \sqrt{1-\epsilon^{2} \sin ^{2}(\tau)} d \tau=\frac{\pi}{2}\left(1-\sum_{i=1}^{\infty} \frac{w_{i}^{2}}{2 i-1} \epsilon^{2 i}\right),
$$

valid for $0 \leq \epsilon<1$. In addition, the series on the right is convergent also for $\epsilon=1$ due to (2.9). Indeed, we have $\frac{w_{i}^{2}}{2 i-1}<\frac{1}{i^{2}}$, which implies the convergence of the series $\sum_{i=1}^{\infty} \frac{w_{i}^{2}}{2 i-1}$.

\footnotetext{
${ }^{5}$ The coefficients of the original Maclaurin series [24] have a visually more complicated form.
} 
Remark 3.1. About fifty years after Maclaurin's book [24], including the series (3.3), Ivory published article [13], where he presented the expansion

$$
\int_{0}^{\frac{\pi}{2}} \sqrt{1-\epsilon^{2} \sin ^{2}(\tau)} \mathrm{d} \tau=\frac{\pi(a+b)}{4 a}\left(1+\sum_{i=1}^{\infty} \frac{w_{i}^{2}}{(2 i-1)^{2}} \lambda^{2 i}\right) \quad\left(\lambda=\frac{a-b}{a+b}\right),
$$

where the series on the right converges slightly faster than the series in (3.3).

Applying (2.9) for the partial sums

$$
\mu_{n}(\epsilon):=\sum_{i=1}^{n} \frac{w_{i}^{2}}{2 i-1} \epsilon^{2 i} \quad(n \in \mathbb{N} \cup\{\infty\}),
$$

we shall estimate the series $\mu_{\infty}(\epsilon)$ figuring in (3.3).

\section{$3.2 \quad$ Approximating $\mu_{\infty}(\epsilon)$}

Using (2.9) we estimate,

$$
\frac{2}{\pi(2 i-1)(2 i+1)}<\frac{w_{i}^{2}}{2 i-1}<\frac{2}{\pi(2 i-1)^{2}} \quad(i \in \mathbb{N}) .
$$

Therefore

$$
\mu_{\infty}(\epsilon) \approx \sum_{i=1}^{\infty} \frac{2 \epsilon^{2 i}}{\pi(2 i-1)(2 i+1)} \quad(0 \leq \epsilon<1) .
$$

This idea produces the next theorem.

Theorem 3.2. We have

$$
\mu_{\infty}(\epsilon)=M_{n}(\epsilon)+\delta_{n}(\epsilon)
$$

where

$$
\begin{gathered}
M_{n}(\epsilon)=A(\epsilon)+B_{n}(\epsilon), \\
A(\epsilon):=\frac{1}{2 \pi}\left[\left(\epsilon-\frac{1}{\epsilon}\right) \ln \left(\frac{1+\epsilon}{1-\epsilon}\right)+2\right] \in\left(0, \frac{1}{\pi}\right), \\
B_{n}(\epsilon):=\sum_{i=1}^{n}\left(w_{i}^{2}-\frac{2}{\pi(2 i+1)}\right) \frac{\epsilon^{2 i}}{2 i-1},
\end{gathered}
$$

and

$$
0<\delta_{n}(\epsilon)<\delta_{n}^{*}(\epsilon):=\frac{2 \epsilon^{2 n+2}}{\pi(2 n+1)^{2}},
$$

valid for any integer $n \geq 1$ and every $0<\epsilon<1$.

The basic function $A(\epsilon)$ is strictly increasing having the range $\left(0, \frac{1}{\pi}\right)$, where $\lim _{\epsilon \downarrow 0} A(\epsilon)=0$ and $\lim _{\epsilon \uparrow 1} A(\epsilon)=\frac{1}{\pi}$. Both sequences, $n \mapsto B_{n}(\epsilon)$ and $n \mapsto \delta_{n}(\epsilon)$, are strictly increasing, for every $\epsilon \in(0,1)$. 
The sequence $n \mapsto M_{n}(\epsilon)$ converges strictly increasingly to $\mu_{\infty}(\epsilon)$, for any $\epsilon \in(0,1)$. Additionally, for every $n \in \mathbb{N}$, the functions $\epsilon \mapsto M_{n}(\epsilon)$ and $\epsilon \mapsto \delta_{n}(\epsilon)$ are strictly increasing on the interval $(0,1)$.

Figure 1 shows, on the left, the graph ${ }^{6}$ of the basic function $A(\epsilon)$, and, on the right, the graphs of the functions $M_{1}^{*}(\epsilon)$ and $\mu_{\infty}(\epsilon)$. As an example, we present $B_{4}^{*}(\epsilon)$ and $\delta_{4}^{*}(\epsilon)$ as follows:

$$
\begin{aligned}
B_{4}^{*}(\epsilon) & =\left(\frac{1}{4}-\frac{2}{3 \pi}\right) \epsilon^{2}+\frac{1}{3}\left(\frac{9}{64}-\frac{2}{5 \pi}\right) \epsilon^{4}+\frac{1}{5}\left(\frac{25}{256}-\frac{2}{7 \pi}\right) \epsilon^{6}+\frac{1}{7}\left(\frac{1225}{16384}-\frac{2}{9 \pi}\right) \epsilon^{8} \\
& \approx 0.037793409 \epsilon^{2}+0.004433682 \epsilon^{4}+0.001342114 \epsilon^{6}+0.000576077 \epsilon^{8}, \\
\delta_{4}^{*}(\epsilon) & \leq \frac{2 \epsilon^{10}}{81 \pi} \leq 0.00786 \epsilon^{10} \quad\left(\epsilon=\sqrt{1-\left(\frac{b}{a}\right)^{2}}\right) .
\end{aligned}
$$
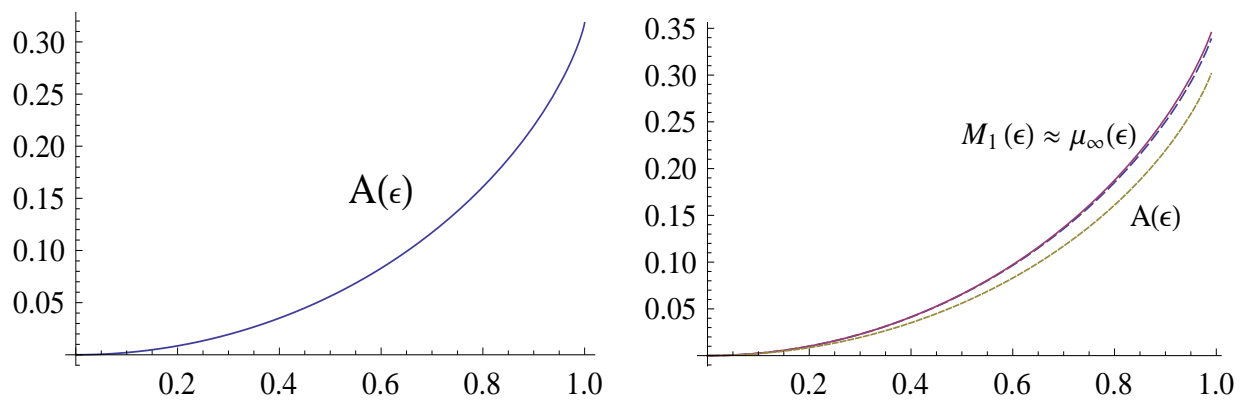

Figure 1: The graph of the basic function $A(\epsilon)$ (left) and the graphs of the functions $M_{1}(\epsilon), \mu_{\infty}(\epsilon)$ and $A(\epsilon)$ (right).

Proof of Theorem 3.2. We have, for $0<\epsilon<1$,

$$
\begin{aligned}
\sum_{i=1}^{\infty} w_{i}^{2} \frac{\epsilon^{2 i}}{2 i-1} & =\sum_{i=1}^{\infty} \frac{2 \epsilon^{2 i}}{\pi(2 i-1)(2 i+1)} \\
& +\sum_{i=1}^{n}\left(\frac{w_{i}^{2}}{2 i-1}-\frac{2}{\pi(2 i-1)(2 i+1)}\right) \epsilon^{2 i}+\delta_{n}(\epsilon)
\end{aligned}
$$

where

$$
\delta_{n}(\epsilon)=\sum_{i=n+1}^{\infty}\left(w_{i}^{2}-\frac{2}{\pi(2 i+1)}\right) \frac{\epsilon^{2 i}}{2 i-1}
$$

\footnotetext{
${ }^{6}$ All the graphics and calculations in this paper are made using the Mathematica [30] computer system.
} 
Moreover, using (2.12), we have

$$
\begin{aligned}
& \sum_{i=1}^{\infty} \frac{2}{\pi(2 i-1)(2 i+1)} \epsilon^{2 i} \\
= & \frac{1}{\pi} \sum_{i=1}^{\infty}\left(\frac{1}{2 i-1}-\frac{1}{2 i+1}\right) \epsilon^{2 i} \\
= & \frac{1}{\pi}\left(\frac{\epsilon}{2} \cdot 2 \sum_{i=1}^{\infty} \frac{\epsilon^{2 i-1}}{2 i-1}-\frac{1}{2 \epsilon} \cdot 2 \sum_{i=1}^{\infty} \frac{\epsilon^{2 i+1}}{2 i+1}\right) \\
= & \frac{1}{\pi}\left[\frac{\epsilon}{2} \ln \left(\frac{1+\epsilon}{1-\epsilon}\right)-\frac{1}{2 \epsilon}\left(\ln \left(\frac{1+\epsilon}{1-\epsilon}\right)-2 \epsilon\right)\right] \\
= & \frac{1}{2 \pi}\left[\left(\epsilon-\frac{1}{\epsilon}\right) \ln \left(\frac{1+\epsilon}{1-\epsilon}\right)+2\right]=A(\epsilon) .
\end{aligned}
$$

Concerning $A(\epsilon)=\frac{1}{2 \pi}(f(\epsilon)+2)$, the function $f(\epsilon):=\left(\epsilon-\frac{1}{\epsilon}\right) \ln \left(\frac{1+\epsilon}{1-\epsilon}\right)(0<\epsilon<1)$ has the derivative $f^{\prime}(\epsilon)=g(\epsilon) / \epsilon^{2}$, where $g(\epsilon)=\left(1+\epsilon^{2}\right) \ln \left(\frac{1+\epsilon}{1-\epsilon}\right)-2 \epsilon$, having the derivative

$$
g^{\prime}(\epsilon)=\frac{2 \epsilon}{1-\epsilon^{2}}\left(2 \epsilon+\left(1-\epsilon^{2}\right) \ln \left(\frac{1+\epsilon}{1-\epsilon}\right)\right)>0 \quad(0<\epsilon<1) .
$$

Thus, $g$ is strictly increasing on $[0,1)$. Consequently, $g(\epsilon)>g(0)=0$, i.e. $f^{\prime}(\epsilon)>0$, for $0<\epsilon<1$. Therefore, $f$ is strictly increasing on $(0,1)$ too. Moreover, using $(2.10)-(2.11)$ with $p=1$, we have

$$
f(\epsilon)=\frac{\epsilon^{2}-1}{\epsilon} \cdot 2\left(\epsilon+\vartheta \cdot \frac{2 \epsilon^{3}}{3\left(1-\epsilon^{2}\right)}\right)=2\left(\epsilon^{2}-1\right)\left(1+\vartheta \cdot \frac{2}{1-\epsilon^{2}} \cdot \frac{\epsilon^{2}}{3}\right),
$$

for some $\vartheta=\vartheta(\epsilon) \in(0,1)$. Hence, $\lim _{\epsilon \downarrow 0} f(\epsilon)=-2$, i.e. $\lim _{\epsilon \downarrow 0} A(\epsilon)=\lim _{\epsilon \uparrow 1} \frac{1}{2 \pi}(f(\epsilon)+2)=0$. In addition, $\lim _{\epsilon \uparrow 1} f(\epsilon)=\lim _{\epsilon \uparrow 1}\left[\frac{\epsilon^{2}-1}{\epsilon} \cdot 2 \ln (1+\epsilon)\right]-\frac{1}{1} \cdot \lim _{h \downarrow 0}(-h \ln (h))=0$, where $h=1-\epsilon^{2}$. Thus, $\lim _{\epsilon \uparrow 1} A(\epsilon)=\lim _{\epsilon \uparrow 1} \frac{1}{2 \pi}(f(\epsilon)+2)=\frac{1}{\pi}$.

According to (3.5), all summands in $B_{n}(\epsilon)$ and $\delta_{n}(\epsilon)$ (see (3.12)) are positive, i.e. the sequences $n \mapsto B_{n}(\epsilon)$ and $n \mapsto \delta_{n}(\epsilon)$ are strictly increasing; consequently the sequence $n \mapsto M_{n}(\epsilon)$ is also strictly increasing, for every $\epsilon \in(0,1)$.

Since all coefficients of the power series $B_{n}(\epsilon)$ and $\delta_{n}(\epsilon)$ (see (3.9) and (3.12)) are positive, due to (3.5), the functions $\epsilon \mapsto M_{n}(\epsilon)$ and $\epsilon \mapsto \delta_{n}(\epsilon)$ are strictly increasing on the interval $(0,1)$, for any $n \in \mathbb{N}$.

According to (3.12) and (3.5), we estimate, for $\epsilon \in(0,1]$,

$$
0<\delta_{n}(\epsilon)<\sum_{i=n+1}^{\infty}\left(\frac{2}{\pi(2 i-1)}-\frac{2}{\pi(2 i+1)}\right) \frac{\epsilon^{2 n+2}}{2 n+1}=\frac{2 \epsilon^{2 n+2}}{\pi(2 n+1)^{2}},
$$

using the telescoping method of summation. 
Example 3.3. Theorem 3.2 is quite useful for an estimate of $\mu_{\infty}(\epsilon)$, consequently for an estimate of the perimeter of an ellipse. For example, for a very flattened ellipse with $q=0.01$ we have $0.99994<\epsilon(q)<0.99995$ where $0.36315<M_{20}(0.99995)<0.36316 \ldots$ and $\delta_{20}^{*}(0.99995)<$ 0.00038. Therefore, $0.36315<\mu_{\infty}(0.99995)<0.36316+0.00038=0.36354$. Thus, to three decimal places, we have $\mu_{\infty}(0.99995)=0.363 \ldots$ Consequently, the perimeter $P(a, b)$ of the corresponding ellipse is given as $P(a, b)=4 a \cdot \frac{\pi}{2}\left(1-\mu_{\infty}(0.99995)\right) \approx 4 a \cdot \frac{\pi}{2}(1-0.363) \approx 4.002 a$ (compare with relations (1.4)).

Remark 3.4. Referring to Abel's theorem on the boundary behavior of a power series, if we continuously extend the domain of the function $A(\epsilon)$ to the closed interval $[0,1]$ by using limits, $A(0):=0$ and $A(1):=\frac{1}{\pi}$, then (3.6), (3.7), (3.9) and (3.10) are all valid also for $\epsilon=0$ and $\epsilon=1$.

Remark 3.5. Alternatively, we can estimate the remainder $r_{n}^{* *}(\epsilon):=\mu_{\infty}(\epsilon)-M_{n}(\epsilon)$ as follows:

$$
\begin{aligned}
r_{n}^{* *}(\epsilon) \leq \sum_{i=n+1}^{\infty} \frac{w_{i}^{2} \epsilon^{2 i}}{2 i-1} & \leq \frac{w_{n+1}^{2} \epsilon^{2 n+2}}{2 n+1} \sum_{j=0}^{\infty} \epsilon^{2 j} \\
& =\frac{w_{n+1}^{2} \epsilon^{2 n+2}}{(2 n+1)\left(1-\epsilon^{2}\right)} \leq \frac{1}{1-\epsilon^{2}} \cdot \frac{2 \epsilon^{2 n+2}}{\pi(2 n+1)^{2}} .
\end{aligned}
$$

This simple method works quite well for $\epsilon$, which "differs enough from 1", but it is useless for $\epsilon$, which is close to 1.

\section{The main result}

Theorem 4.1. For every $n \in \mathbb{N}$, the perimeter $P(a, b)$ of an ellipse having semi-major and semiminor axes, $a$ and $b$, the aspect ratio $q=q(a, b):=b / a$, and the eccentricity $\epsilon=\epsilon(a, b):=\sqrt{1-q^{2}}$, the $n$-th approximation $Q_{n}(a, b) \approx P(a, b)$,

$$
Q_{n}(a, b):=2 \pi a\left(1-M_{n}(\epsilon)\right)=2 \pi a\left(1-A(\epsilon)-B_{n}(\epsilon)\right)
$$

has the relative error,

$$
\frac{P(a, b)-Q_{n}(a, b)}{P(a, b)}=: \rho_{n}(q) \quad\left(q=q(a, b)=\left(\frac{b}{a}\right)^{2}\right)
$$

estimated as

$$
-\frac{1}{(2 n+1)^{2}} e^{-q^{2}(n+1)} \leq-\frac{\left(1-q^{2}\right)^{n+1}}{(2 n+1)^{2}}=: \rho_{n}^{*}(q) \leq \rho_{n}(q) \leq 0 .
$$

Here, $A(\epsilon)$ and $B_{n}(\epsilon)$ are defined in Theorem 3.2 and we have $B_{n+1}(\epsilon)=B_{n}(\epsilon)+\left(w_{n+1}^{2}-\frac{2}{\pi(2 n+3)}\right) \frac{\epsilon^{2 n+2}}{2 n+1}$, for $n \in \mathbb{N}$ and $0 \leq \epsilon \leq 1$. 
Proof. Thanks to (1.1), (1.2), (1.4) and (3.3), we estimate

$$
\begin{aligned}
-\frac{P(a, b)-Q_{n}(a, b)}{P(a, b)} & =-\frac{2 \pi a\left(1-M_{n}(\epsilon)-\delta_{n}(\epsilon)\right)-2 \pi a\left(1-M_{n}(\epsilon)\right)}{P(a, b)} \\
& \stackrel{(1.4)}{<} \frac{2 \pi a \delta_{n}(\epsilon)}{4 a} \leq \frac{\pi \delta_{n}(\epsilon)}{2}<\frac{\pi}{2} \cdot \frac{2 \epsilon^{2 n+2}}{\pi(2 n+1)^{2}}=\frac{\epsilon^{2 n+2}}{(2 n+1)^{2}},
\end{aligned}
$$

where, considering the convexity of the exponential function or, referring to [16, (6a)] with $\varepsilon=q^{2}$ and $h=-q^{2}$, we have

$$
\epsilon^{2 n+2}=\left(1-q^{2}\right)^{n+1} \leq e^{-q^{2}(n+1)} \quad(0 \leq q<1) .
$$

Figures 2-3 show, for several values of $n$, the graphs of actual relative errors $-\rho_{n}(q)=$ $\left[\mu_{\infty}(\epsilon(q))-M_{n}(\epsilon(q))\right] /\left[1-\mu_{\infty}(\epsilon(q))\right]$ (left) together with their upper bounds $-\rho_{n}^{*}(q)$ (right).
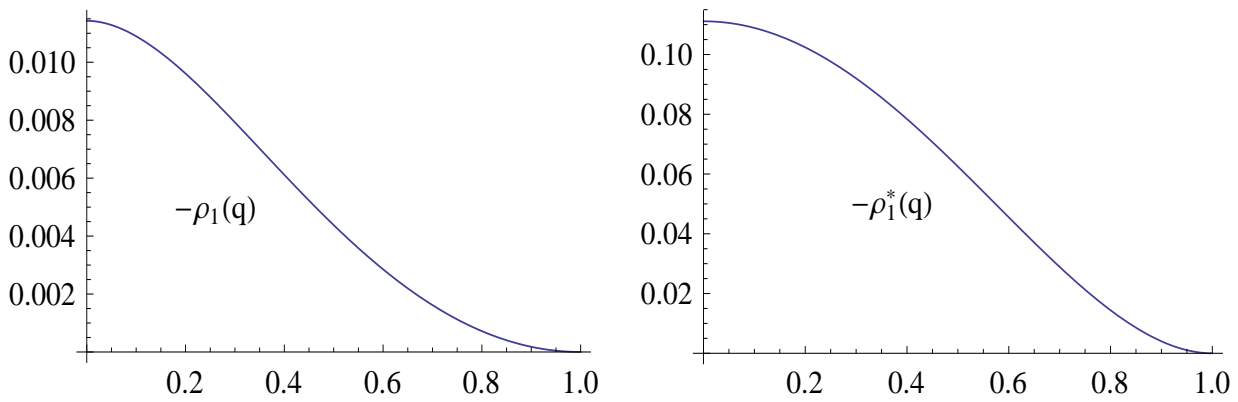

Figure 2: The graphs of the functions $q \mapsto-\rho_{1}(q)$ and $q \mapsto-\rho_{1}^{*}(q)$.
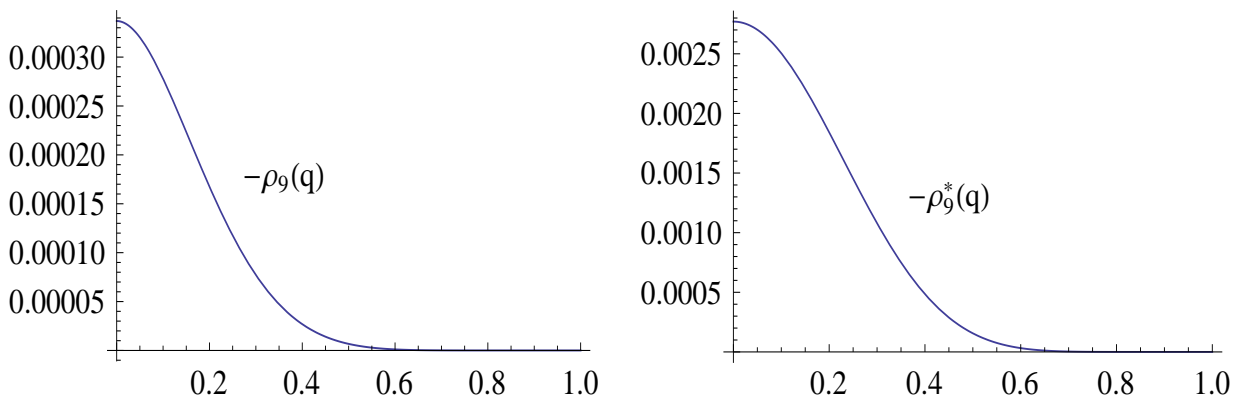

Figure 3: The graphs of the functions $q \mapsto-\rho_{9}(q)$ and $q \mapsto-\rho_{9}^{*}(q)$.

Table 1 additionally confirms the usefulness of the derived formula.

Conclusion. The article demonstrates that with the help of 277 years old Maclaurin series the perimeter of an ellipse can be accurately estimated, even if an ellipse flattens into a line segment. This is done only by elementary means, not using complex analysis or elliptical integral theory, neither arithmetic-geometric means nor hypergeometric functions. 


\begin{tabular}{|c||c|c|c|c|c|c|}
\hline$q$ & 0.00001 & 0.1 & 0.2 & 0.3 & 0,4 & 0,5 \\
\hline$-\rho_{20}(q)$ & $<8 \cdot 10^{-5}$ & $<6 \cdot 10^{-5}$ & $<2 \cdot 10^{-5}$ & $<5 \cdot 10^{-6}$ & $<6 \cdot 10^{-7}$ & $<4 \cdot 10^{-8}$ \\
\hline$-\rho_{20}^{*}(q)$ & $<6 \cdot 10^{-4}$ & $<5 \cdot 10^{-4}$ & $<3 \cdot 10^{-4}$ & $<9 \cdot 10^{-5}$ & $<2 \cdot 10^{-5}$ & $<2 \cdot 10^{-6}$ \\
\hline
\end{tabular}

Table 1: The actual error $\rho_{20}(q)$ and the a priori estimated error $\rho_{20}^{*}(q)$.

\section{References}

[1] S. Adlaj, An eloquent formula for the perimeter of an ellipse, Notices of the AMS 59 (2012), no. 8, 1094-1099.

[2] G. Almkvist and B. Berndt, Gauss, Landen, Ramanujan, the arithmetic-geometric mean, ellipses, $\pi$, and the Ladies Diary, Amer. Math. Monthly 95 (1988), 585-608.

[3] B. W. Barnard, K. Pearce and L. Schovanec, Inequalities for the perimeter of an Ellipse, J. Math. Anal. Appl. 260 (2001), 295-306.

[4] C.-P. Chen and F. Qi, Best upper and lower bounds in Wallis' inequality, Journal of the Indonesian Mathematical Society 11 (2005), no. 2, 137-141.

[5] C.-P. Chen and F. Qi, The best bounds in Wallis' inequality, Proc. Amer. Math. Soc. 133(2005), 397-401.

[6] C.-P. Chen and F. Qi, Completely monotonic function associated with the gamma functions and proof of Wallis' inequality, Tamkang Journal of Mathematics 36 (2005), no. 4, 303-307.

[7] V. G. Cristea, A direct approach for proving Wallis' ratio estimates and an improvement of Zhang-Xu-Situ inequality, Studia Univ. Babeș-Bolyai Math. 60 (2015), 201-209.

[8] J.-E. Deng, T. Ban and C.-P. Chen;Sharp inequalities and asymptotic expansion associated with the Wallis sequence, J. Inequal. Appl., (2015), 2015:186.

[9] S. Dumitrescu, Estimates for the ratio of gamma functions using higher order roots, Studia Univ. Babeș-Bolyai Math. 60 (2015), 173-181.

[10] S. Guo, J.-G. Xu and F. Qi,Some exact constants for the approximation of the quantity in the Wallis' formula, J. Inequal. Appl., (2013), 2013:67.

[11] S. Guo, Q. Feng, Y.-Q. Bi and Q.-M. Luo, A sharp two-sided inequality for bounding the Wallis ratio, J. Inequal. Appl., (2015), 2015:43.

[12] B.-N. Guo and Feng Qi, On the Wallis formula, International Journal of Analysis and Applications 8 (2015), no. 1, 30-38. 
[13] J. Ivory, A new series for the rectification of the ellipsis; together with some observations on the evolution of the formula $\left(a^{2}+b^{2}-2 a b \cos \phi\right)^{n}$, Trans. Royal Soc. Edinburgh 4 (1796), $177-190$.

[14] A. Laforgia and P. Natalini, On the asymptotic expansion of a ratio of gamma functions, J. Math. Anal. Appl. 389 (2012), 833-837.

[15] V. Lampret, The Euler-Maclaurin and Taylor Formulas: Twin, Elementary Derivations, Math. Mag. 74 (2001), No. 2, pp. 109-122.

[16] V. Lampret, Wallis' Sequence Estimated Accurately Using an Alternating Series, J. Number. Theory. 172 (2017), 256-269.

[17] V. Lampret, A Simple Asymptotic Estimate of Wallis Ratio Using Stirlings Factorial Formula, Bull. Malays. Math. Sci. Soc. (2018), doi.org/10.1007/s40840-018-0654-5.

[18] C. E. Linderholm and A. C. Segal, An Overlooked Series for the Elliptic Perimeter, Mathematics Magazine, 68(1995)3, 216-220.

[19] C. Mortici, Sharp inequalities and complete monotonicity for the Wallis ratio, Bull. Belg. Math. Math. Soc. Simon Stevin, 17 (2010), pp. 929-936.

[20] C. Mortici, New approximation formulas for evaluating the ratio of gamma functions, Math. Comput. Modelling 52 (2010), pp. 425-433.

[21] C. Mortici, A new method for establishing and proving new bounds for the Wallis ratio, Math. Inequal. Appl. 13 (2010), 803-815.

[22] C. Mortici, Completely monotone functions and the Wallis ratio, Appl. Math. Lett. 25 (2012), $717-722$.

[23] C. Mortici and V. G. Cristea, Estimates for Wallis' ratio and related functions, Indian J. Pure Appl. Math. 47 (2016), 437-447.

[24] C. A. Maclaurin, A treatise of fluctions in two books, Vol.2, T. W. and T. Ruddimans, Edinburgh 1742 .

[25] F. Qi and C. Mortici, Some best approximation formulas and the inequalities for the Wallis ratio, Appl. Math. Comput. 253 (2015), 363-368.

[26] , F. Qi, An improper integral, the beta function, the Wallis ratio, and the Catalan numbers, Problemy Analiza-Issues of Analysis 7 (25) (2018), no. 1, 104-115.

[27] J.-S. Sun and C.-M. Qu, Alternative proof of the best bounds of Wallis' inequality, Commun. Math. Anal. 2 (2007), 23-27. 
[28] M. B. Villarino, A Direct Proof of Landen's Transformation, arXiv:math/0507108v1 [math.CA].

[29] M. B. Villarino, Ramanujan's inverse elliptic arc approximation, Ramanujan J., 34 (2014), no. $2,157-161$.

[30] S. Wolfram, Mathematica, Version 7.0, Wolfram Research, Inc., 1988-2009.

[31] X.-M. Zhang, T. Q. Xu and L. B. Situ Geometric convexity of a function involving gamma function and application to inequality theory, J. Inequal. Pure Appl. Math. 8 (2007) 1, art. 17, $9 \mathrm{p}$. 